Titulo do Trabalho

\title{
AVALIAÇÃO DA QUALIDADE DA ÁGUA DA LAGOA MAIOR, TRÊS LAGOAS/MS, UTILIZANDO MACROINVERTEBRADOS BENTÔNICOS COMO BIOINDICADORES.
}

\author{
Nome do Autor (a) Principal \\ Diego Emanuel Ferraz
}

Nome (s) do Coautor (a) (s)

Rodrigo Martins dos Santos; Maria Elisa Rebustini.

Nome (s) do Orientador (a) (s)

Dra Maria Elisa Rebustini

Instituição ou Empresa

Universidade Federal de Mato Grosso do Sul

Instituição (s) de Fomento

Universidade Federal de Mato Grosso do Sul

E-mail de contato

piracicabadiego@gmail.com

Palavras-chave

Bioindicadores, Lagoa, Macroinvertebrados bentônicos.

\section{INTRODUÇÃO}

Os macroinvertebrados constituem um dos bioindicadores mais viáveis na avaliação da qualidade das águas de sistemas continentais. Diversos pesquisadores, como Monaghan e Soares. (2012); Al-Shami et al. (2011); König et al. 2008; Yu, L. et 
al., (2004); Callisto et al.(2001) estudaram a qualidade da água utilizando o índice BMWP (Biological Monitoring Work Party Score System) que avalia as condições da água, utilizando a pontuação atribuída às famílias de macroinvertebrados chaves, com respostas já conhecidas frente às alterações em seu habitat. Este índice foi adaptado às condições brasileiras por pesquisadores da Secretaria do Meio Ambiente do Paraná, incluindo em suas avaliações novas famílias encontradas em águas brasileiras e também bem conhecidas, quanto à sua resposta às variações em seu habitat (IAP, 2013).

\section{OBJETIVO GERAL}

Avaliar a qualidade da água, utilizando macroinvertebrados como bioindicadores.

\section{OBJETIVOS ESPECÍFICOS}

O objetivo deste trabalho foi avaliar a qualidade da água da Lagoa do Maior do município de Três Lagoas/MS, utilizando macroinvertebrados bentônicos como bioindicadores dos quais foram aplicados ao índice BMWP fornecido pela Secretaria do Meio Ambiente e Recursos Hídricos do Paraná.

\section{METODOLOGIA}

O presente estudo foi realizado na lagoa Maior pertencente ao complexo lacustre do município de Três Lagoas-MS no mês de outubro de 2013. Foram demarcados 4 pontos (PO1, PO2, PO3 e PO4) e para a coleta dos dados foi utilizada rede de coleta de macroinvertebrados bentônicos nas áreas mais rasas que são as preferidas para nidificação de insetos aquáticos. As amostras foram encaminhadas para o Laboratório de Zoologia do CPTL/UFMS, para triagem dos macroinvertebrados obtidos.

Após a triagem, os macroinvertebrados bentônicos foram identificados até menor nível taxonômico possível utilizando chaves de identificação de Mugnai et al. (2009). O índice utilizado para avaliar a qualidade da água foi o BMWP (Biological Monitoring Work Party Score System) que por sua vez, é uma adaptação referente as condições brasileiras feita por pesquisadores da Secretaria do Meio Ambiente do 
Paraná (IAP) conforme quadro 1. Esse índice atribui valores para cada família com base na sua tolerância ao impacto, e os valores variam entre 1 e 10 são atribuídos de acordo com a sensibilidade das famílias a poluentes, sendo que famílias sensíveis a altos níveis de poluentes recebem valores mais altos, enquanto famílias tolerantes recebem valores mais baixos.

Quadro 1 - Soma dos índices atribuídos as famílias encontradas e o seu significado, em relação a qualidade da água, proposto pela SECRETARIA DO MEIO AMBIENTE DO PARANÁ.

\begin{tabular}{|c|c|c|l|l|}
\hline CLASSE & QUALIDADE & VALOR & SIGNIFICADO & COR \\
\hline I & ÓTIMA & $>150$ & Águas muito limpa (águas pristinas) & LILÁS \\
\hline II & BOA & $121-150$ & $\begin{array}{l}\text { Águas limpas, não poluídas ou sistema } \\
\text { perceptivelmente não alterado. }\end{array}$ & $\begin{array}{l}\text { AZUL } \\
\text { ESCURO }\end{array}$ \\
\hline III & ACEITÁVEL & $101-120$ & $\begin{array}{l}\text { Águas muito pouco poluída, ou sistema já com um } \\
\text { pouco de alteração. }\end{array}$ & $\begin{array}{l}\text { AZUL } \\
\text { CLARO }\end{array}$ \\
\hline IV & DUVIDOSA & $61-100$ & São evidentes efeitos moderados de poluição & VERDE \\
\hline V & POLUÍDA & $36-60$ & Águas contaminadas ou poluídas (sistema alterado) & AMARELO \\
\hline VI & MUITO POLUÍDA & $16-35$ & Águas muito poluídas (sistema muito alterado). & LARANJA \\
\hline VII & $\begin{array}{c}\text { FORTEMENTE } \\
\text { POLUÍDA }\end{array}$ & $<16$ & $\begin{array}{l}\text { Águas fortemente poluídas (sistema fortemente } \\
\text { alterado). }\end{array}$ & VERMELHO \\
\hline
\end{tabular}

Fonte: (IAP, 2013) 
Figura 1: Pontos de coleta para avaliação de macroinvertebrados bentônicos na Lagoa Maior de Três Lagoas/MS

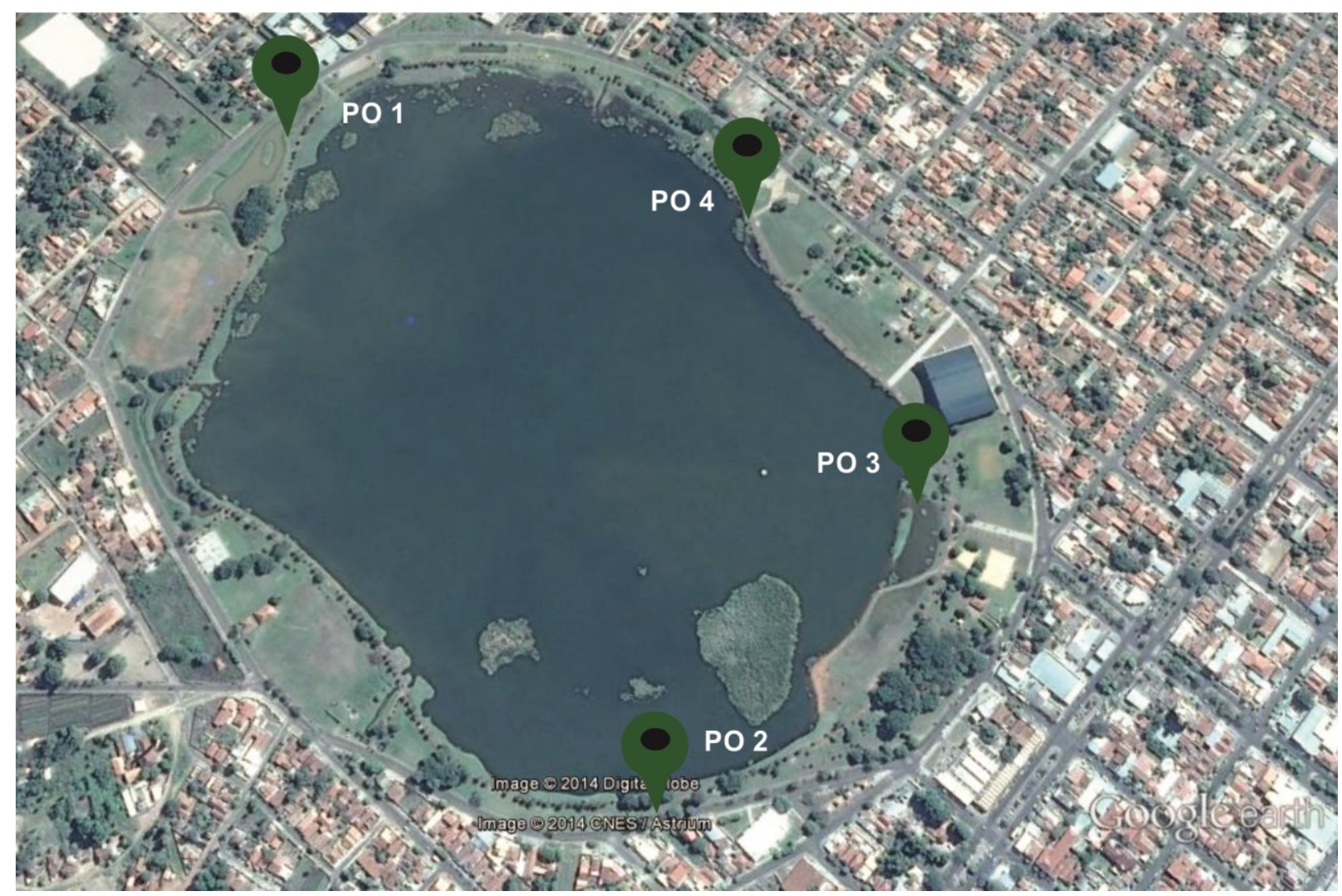

Em PO1 (Ponto1) caixa de contenção de águas pluviais; PO2 (Ponto 2) próximo a biblioteca; PO3 (ponto 3) ponto próximo a academia da Terceira Idade; $\mathrm{PO} 4$ (ponto 4) próximo à praça de exercício.

Fonte: Extraído e modificado dos sites Google ® Earth (๑Google - Imagens @2013 Digital Globe).

\section{RESULTADOS E DISCUSSÕES}

Foram encontrados no total 698 espécimes, dos quais 628 foram identificadas até nível de família, e 70 espécimes identificados até o nível de ordem. As famílias identificadas são apresentadas a seguir: Thiaridae (357), Planorbidae (129), Physidae(26), Ampularidae (23), Hirudidae(16), Psychodidae(02), Gyrinidae (02), Corixidae (22), Aeshnidae (03), Odontoceridae (04), Tabanidae (02), Vellidae (01), Leptohyphidae (01), Chironomidae (24), Culicidae (01), Ancilidae (03), Haliplidae (01), Hydroptilidae (01), Noteridae (02), Baetidae (04), Elmidae (02) ,Belostomatidae (02); os indivíduos pertencente as Ordens: Oligochaeta (60) Lepidoptera(01), Bivalvia(06), Hidracarina(03). 
Considerando todos os pontos estudados, verificou-se que a família Thiaridae apresentou taxas populacionais mais elevadas em todos os pontos. A presença desta família se destaca, uma vez que inclui o gênero Melanoides considerado exótico sendo documentado no Brasil pela primeira vez em 1967 e, atualmente, já foi registrado em 17 estados brasileiros (THIENGO et al.,2001). De acordo com Fernandez et al.2003 e Bógea et al. (2005), a família Thiaridae além de ser invasora pode vir hospedar parasitas importantes para a medicina humana, como a paragonimose e clonorquiose, endêmicas da Ásia Oriental. As famílias Physidae e Planorbidae também foram amostradas em todos os pontos.

A quantidade de indivíduos da família Thiaridae (figura 2), caracterizou uma rápida expansão do grupo com competição com as outras espécies nativas, sendo sua característica marcante de colonizar áreas impactadas por atividades antrópicas, principalmente locais de águas rasas, com substrato de granulometria fina e rico em detritos orgânicos Pointier et al. (1993); De Marco Junior. (1999); Martins-Silva \& Barros. (2001). Entretanto, apesar das características serem bem próximas, é preciso um estudo mais aprofundado deste grupo, para confirmação da espécie Melanoides tuberculatus sendo uma espécie partenogenética com certo grau de resistência à poluição e, portanto, dispersa-se rapidamente, encobrindo quase totalmente o sedimento e competindo diretamente com as espécies nativas (ROSA et al., 2010).

Figura 2: Indivíduos da família Thiaridae amostrados em todos os pontos avaliados.

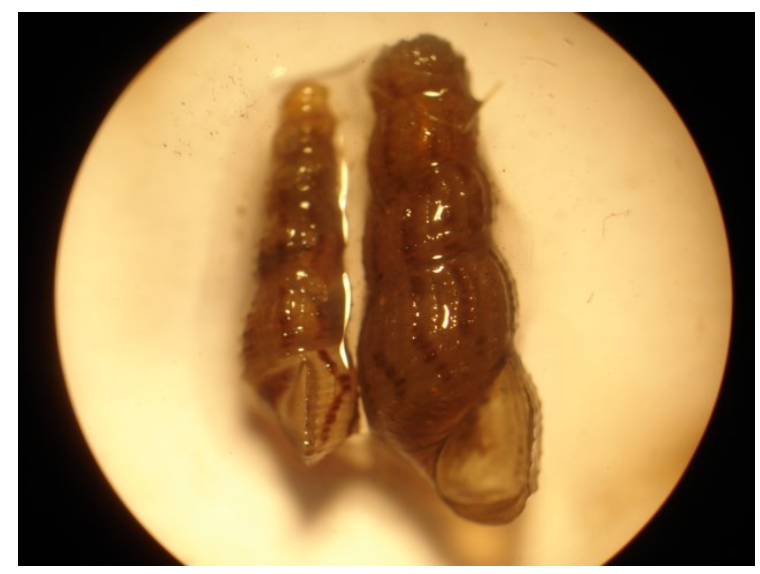

Fonte: Laboratório de Zoologia/CPTL/UFMS, 2013 
De maneira geral, a ordem Oligochaeta também esteve em todos os pontos indicando que o sistema se encontra impactado; em vista que altas densidades dessa ordem podem evidenciar o elevado teor de matéria orgânica no ambiente (MARQUES et al., 1999), que os tornam eficientes indicadores de degradação ambiental. Com relação aos Oligochaeta estes são um dos macroinvertebrados bentônicos de grande importância, pois sua presença tem sido reportada por estarem presentes em ambientes organicamente poluídos (MILBRINK, 1994; LANG, 1997; 1998); com isso a presença deste.

Para a avaliação da qualidade da água da lagoa, as pontuações totais do índice BMWP referentes a cada ponto estudado (Tabela 1), foram comparadas com a tabela proposta pela Secretaria do Meio Ambiente do Paraná (Quadro1), que correlaciona estes valores a cinco graus diferenciados de qualidade da água.

De acordo com a pontuação BMWP, todos os pontos amostrados se encontraram no valor de 36 a 60, logo foi visto que a qualidade da água da Lagoa encontra-se poluída. Estes resultados mostram que existe fatores influenciando a qualidade da água da Lagoa Maior.

Tabela 1: Pontuações totais obtidas pela soma dos índices BMWP atribuídos a cada família dos pontos amostrados

\begin{tabular}{lll}
\hline Pontos de Coleta & Pontuação BMWP & ${ }^{* *}$ Cor \\
\hline PO1 & 48 \\
PO2 & 58 \\
PO3 & 46 \\
PO4 & 60 \\
\cline { 2 - 2 } As cores representam a qualidade da água em cada ponto
\end{tabular}

Fonte: Secretaria Do Meio Ambiente Do Paraná. (IAP, 2013)

\section{CONSIDERAÇÕES FINAIS}

Os baixos valores referentes ao índice BMWP indicam que o sistema apresenta-se impactado bem como a qualidade da água encontra-se poluída. 
Já o ponto PO4 obteve elevados índices de indivíduos da família Thiaridae indicando assim, possíveis alterações no local que precisam ser confirmadas com estudos mais aprofundados.

Os resultados obtidos nesta pesquisa indicam que os indivíduos das famílias Thiaridae devem ser mais estudados, com o objetivo de identificar gêneros presentes na lagoa, dando ênfase no gênero Melanoides, pois além de seu caráter invasor, se destaca também pela sua importância médica.

\section{REFERÊNCIAS}

AL-SHAMI, S; RAWI, C. S. M.; AHMAD, A. H.; HAMID, S. A.; NOR, S. A. M. Influence of agricultural, industrial, and anthropogenic stresses on the distribution and diversity of macroinvertebrates in Juru. Ecotoxicol Environ Saf, v. 74, n. 5, p. 1195-1202, 2011.

BÓGEA, T.; CORDEIRO, F.M. \& GOUVEIA, J. S., 2005. Melanoides tuberculatus(Gastropoda: Thiaridae) as intermediate host of Heterophyidae (Trematoda: Digenea) in Rio de Janeiro metropolitan área, Brazil. Ver. Inst. Med. Trop. S. Paulo.

CALLISTO, M.; MORETTI, M.; GOULART. Macroinvertebrados bentônicos como ferramenta para avaliar a saúde de riachos. RBRH - Revista Brasileira de Recursos Hídricos, v. 6, n. 1, p. 71-82, 2001.

CHAPMAN \& HALL, 1993. The abatement of eutrophication in Lake Neuchâtel. Aquat. sci, Basel, v. 61, p. 206-214,1998.

De MARCO JUNIOR, P. Invasion by the introduced aquatic snail Melanoides tuberculata (Müller, 1774) (Gastropoda: Prosobranchia: Thiaridae) of the Rio Doce State Park, Minas Gerais, Brazil. Stud. Neotrop. Fauna Environ., 34: 186-189, 1999.

FERNANDEZ, M. A., THIENGO, S. C. \& SIMONE, L.R. L., 2003. Distribution of the introduced freshwater snail Melanoides tuberculatus (Gastropoda: Thiaridae) in Brazil.

IAP. Avaliação da Qualidade da Água Através dos Macroinvertebrados Bentônicos - Índice BMWP. Secretaria do Meio Ambiente do Paraná <http://www.meioambiente.pr.gov.br/modules/conteudo/conteudo.php?conteudo=91>.(Acesso nos períodos de execução do trabalho).

KÖNIG, R.; SUZIN, C. R. H.; RESTELLO, R. M.; HEPP, L. U. Qualidade das águas de riachos da região norte do Rio Grande do Sul (Brasil) através de variáveis físicas, químicas e biológicas. Pan-American Journal of Aquatic Sciences, v. 3, n. 1, p. 84-93, 2008.

LANG, C. Constrasting responses of oligochaeta (Annelida) and chironomids (Diptera) to the abatement of eutrophication in Lake Neuchâtel. Aquat. sci, Basel, v. 61, p. 206-214, 1998.

MARQUES, M. G. S. M.; FERREIRA, R. L. \& BARBOSA, F. A. R. A comunidade de Macroinvertebrados aquáticos e características limnológicas das lagoas carioca e da barra, Parque Estadual do Rio Doce, MG. Revista Brasileira de Biologia 59(2): p. 203- 210.1999. 
MARTINS-SILVA, M. \& BARROS, M. Occurence and distribution of freshwater molluscs in the Riacho Fundo Creek Basin, Brasília, Brazil. Rev. Biol. Trop., 49(3): 864-870.

MILBRINK, G. Oligochaetes and pollution in two deep Norwegian lakes. Hydrobiologia 278: p. 213-222. 1994

MONAGHAN, K. A.; SOARES, A. M. V. M. Bringing new knowledge to an old problem: Building a biotic index from lotic macroinvertebrate traits. Ecological Indicators, v. 20, p. 213-220, 2012.

MUGNAI, R.; BAPTISTA, D.F.; NESSIMIAN,J.L.Manual de identificação de macroinvertebrados do estado do Rio de Janeiro.Rio de Janeiro: Technical Books,2009.

POINTIER, J.P.; THALER, L.; PERNOT, A.F. \& DELAY, B. Invasion of the Martinique island by the parthenogenetic snail Melanoides tuberculata and the succession of morphos. Acta Ecol., 14: 3342, 1993.

ROSA, L. C.; SOUTO; BRITO ,M. F. G. Ocorrência do molusco exótico invasor Melanoides tuberculatus na Lagoa Azul, APA Litoral Sul, Sergipe, Brasil. In: encontro de recursos hídricos em sergipe, 3., 2010, Sergipe. Resumo . Sergipe: Universid Ade Federal de Sergipe, 2010. p. 21 - 27.

ROSENBERG, D. M.; RESH, V. H. Freshwater biomonitoring and benthic macroinvertebrates.

THIENGO, S. C. et al. Freshwater snails and schistosomiasis mansoni in the State of Rio de Janeiro, Brazil: I - Metropolitan mesoregion. Mem. Inst. Oswaldo Cruz, v. 96, p. 177-184, 2001.

YU, L. ; VERMAAT, J. E., RUYTER, E. D. de; KRUIJF, H. A. M. de The modification and the application of biomonitoring ISO-BMWP method of macrofauna in the river pollution evaluation in China . Acta Sci. Nat. Univ. Sunyatseni/Zhongshan Daxue Xuebao, v. 43, n. 4, p. 102-105, 2004. 